\title{
Implementation of Biological Sources of Energy in the System of "Smart Clothes"
}

\author{
Alexander Molnar ${ }^{1}$, Vitaly Gerasimov ${ }^{2, *}$, Anna Badidová ${ }^{3}$ \\ 1 Uzhhorod National University, Department of the Physics of Semiconductors, Uzhhorod, Ukraine \\ 2 Mukachevo State University, Department of Light Industry, Mukachevo, Ukraine \\ 3Technical University of Košice, Department of Process and Environmental Engineering, Košice, Slovakia
}

\begin{abstract}
The article is devoted to problems of powering embedded devices of "smart clothes". The growth rates of interest in bioenergy energy sources and their variety in the world are shown. The authors analysed the technical and energy indicators of the human body as an energy source for various types of portable devices. The technical characteristics of possible energy converters, which are suitable for this application, are described. Schematic solutions for powering portable devices based on ready solutions are proposed.
\end{abstract}

Keywords: embedded devices; human energetic; harvesting technology.

\section{Introduction}

Nowadays in the market there are no items representing practical and successful results in the sphere of "smart clothes" development. Although the ideas concerning creation of such clothes have been here for a while and there is a solid theoretical possibility to sew "smart" outfits, not so many electronic jackets and sweaters have been developed so far, let alone the ones that sell well. The reasons for this situation are numerous, the major one being the absence of efficient, reliable and functional energy cells for portable systems.

Under the current conditions the power consumption of electronic components (analogue-to-digital converter, microcontrollers as well as supplementary digital and analogue microcircuits), which are an integral part of any mobile system including "smart clothes", is so insignificant that there arises possibility to implement alternative sources of electric energy (i.e. piezoelectric [1], thermoelectric, photoelectric etc.). However, this list can be complemented with new unconventional types of energy sources, among which bioenergy can be singled out. The authors consider the issue mentioned above within the frame of energy procurement for the system of "smart clothes".

\section{Human Energetics}

The human body is a huge energy reservoir. An average person weighing as much as $68 \mathrm{~kg}$ with the fat reserve of $15 \%$ contains the amount of energy sufficient to supply a four-room apartment with electricity for the period of 2.67 days [2]. It demonstrates how well our body operates as a system of potential energy reserve. The amounts of energy used up by people during various types of activity are presented in Figure 1. It shows that even during sleeping a person dissipates substantial amount of power, which can be compared with the power consumption of an old (CRT) computer monitor. The work of energy transformation systems should not have a big impact on the bearer (a person). Moreover, it has to be taken into account that the coefficient of efficiency characterizing the best energy transformation systems is still far from 
desired (<50\%).

In order to estimate the maximal power derived from human activity without causing any significant discomfort to a person, it is necessary to consider the main energy sources of a human body. They comprise body heat generation, exhalation, blood pressure, fingers motion, arm and leg motion, walking and running, chemical energy received through nutrition etc. The main of them are shown in Figure 1 indicating the maximal power of each one.

Table 1: Energy consumption for certain types.

\begin{tabular}{|l|l|l|}
\hline Activity & Kilocal/hr & Watts \\
\hline Sleeping & 70 & 81 \\
\hline Lying quietly & 80 & 93 \\
\hline Sitting & 100 & 116 \\
\hline Standing at ease & 110 & 128 \\
\hline Conversation & 110 & 128 \\
\hline Eating meal & 110 & 128 \\
\hline Strolling & 140 & 163 \\
\hline Driving car & 140 & 163 \\
\hline $\begin{array}{l}\text { Playing violin or } \\
\text { piano }\end{array}$ & 140 & 163 \\
\hline Housekeeping & 150 & 175 \\
\hline Carpentry & 230 & 268 \\
\hline Hiking, 4 mph & 350 & 407 \\
\hline Swimming & 500 & 582 \\
\hline $\begin{array}{l}\text { Mountain clim- } \\
\text { bing }\end{array}$ & 600 & 698 \\
\hline $\begin{array}{l}\text { Long distance } \\
\text { run }\end{array}$ & 900 & 1,048 \\
\hline Sprinting & 1400 & 1,630 \\
\hline
\end{tabular}

Considering pure heat energy dissipated by a person, the values of it are certainly much lower (Figure 2) than those of motion. Apart from that, it is necessary to take into account the temperature of the environment. As can be seen from Figure 2, temperature converters have to be located either on the head (hat) or in the area of the neck/chest.

Apart from the energy sources presented in Figure 3, there are other ways to produce electrical energy, which have not been implemented yet, both technologically and technically, and are insufficiently explored. Each of these methods has

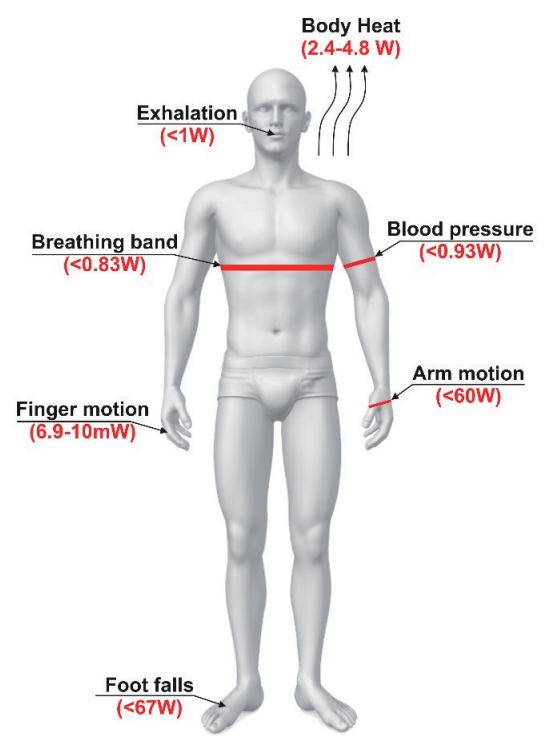

Fig. 1: Main energy sources in human body of human activity. (maximal power is indicated in brackets).

Table 2: Power dissipated as heat from the human different room temperatures [3] body during different activities [4].

\begin{tabular}{|l|l|l|l|}
\hline \multirow{2}{*}{ Activity } & \multicolumn{3}{|c|}{ Power (W) } \\
\cline { 2 - 5 } & Total & Sensible & Latent \\
\hline Seated at rest & 100 & 60 & 40 \\
\hline Seated light work (writing) & 120 & 65 & 55 \\
\hline Seated eating & 170 & 75 & 95 \\
\hline Walking at 1.5 m/sec & 305 & 100 & 205 \\
\hline Heavy work (lifting) & 465 & 165 & 300 \\
\hline Athletics & 525 & 185 & 340 \\
\hline $\begin{array}{r}\text { Core temp } \\
37^{\circ} \mathrm{C} \\
36^{\circ} \mathrm{C} \\
34^{\circ} \mathrm{C} \\
32^{\circ} \mathrm{C} \\
31^{\circ} \mathrm{C} \\
28^{\circ} \mathrm{C}\end{array}$ \\
\hline
\end{tabular}

Fig. 2: The skin temperature map.

the highest possible power index per one person. Many of them can be combined technologically, technically as well as in terms of circuit- and systems 
engineering. Modern electronic industry has already been producing specialized microcircuit chips for "collection", transformation and distribution of energy derived from different sources (so called energy harvesters). LINEAR Technology Company (Figure 3) can serve as an example of such a manufacturer. Moreover, we would like to draw your attention to the brand new methods implemented as energy cells in order to get energy, for instance, biochemical sources. The latest achievements in the fields of micro- and nano-biology demonstrate the first positive results and give hopes for their practical implementation.

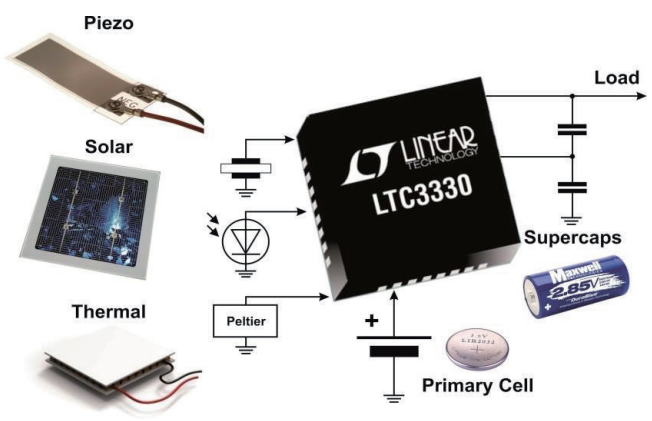

Fig. 3: Implementation of various energy sources for "smart clothes" power system.

Sources of energy based on internal potentials of the body. It is widely known that human muscles are guided by electric signals and are themselves the sources of potentials which can be implemented for diagnostics (electromyography), treatment (electroacupuncture) and generation of electric current. Unfortunately, the muscle potential oscillations amplitude, as a rule, does not exceed several millivolts, whereas their duration $-20 \div 25$ milliseconds. Until recently there have not been any possibilities to implement such small powers efficiently. However, the situation has changed substantially. In his work [5] the author describes a system implementing Endocochlear potential - positive voltage with the quantity of $80-100 \mathrm{mV}$, which appears in cochlear endolymphatic cavities of the inner ear.

This biogenerator produces just 1.12 nWatt of electric energy, but it is sufficient for powering the system as it transmits the data from the built-in sensor to the system of telemetry with the interval of $40-360$ sec. at frequency $2.4 \mathrm{GHz}$.

In future analogous power systems can be implemented for implanted Blue Tooth headsets, hearing devices etc.

\section{Bio-batteries implementing glucose of human blood or sweat}

Unlike secondary human activities such as movement and, correspondingly, muscular biopotentials, live organism energy sources can be implemented straightforwardly and much more efficiently. Glucose is the main energy source of the body as its energy density is very high. For instance, glucose burning power amounts to $15.5 \mathrm{MJ} / \mathrm{kg}$. Glucose can release up to 3,574 A-hour/kg, which is 85 times more than the energy emitted by lithiumion batteries (42 A-hour/kg) [6]. Therefore, there have been active researches to discover artificial

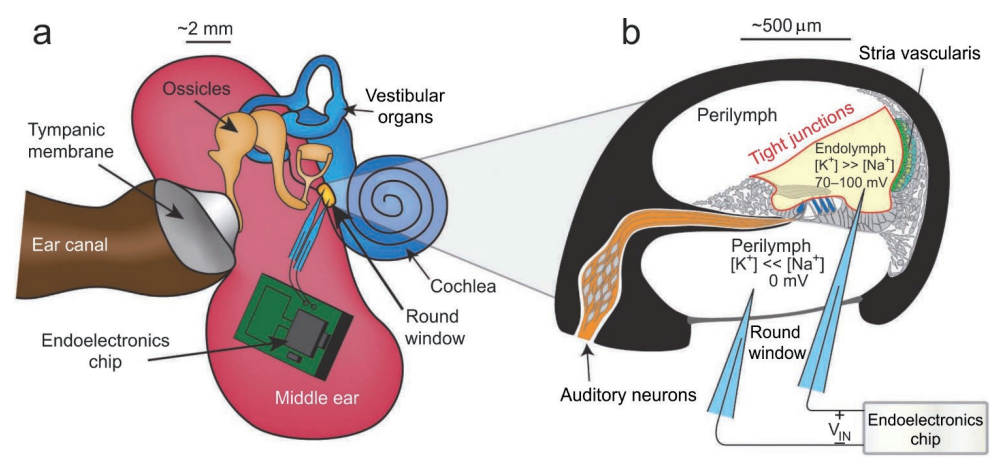

Fig. 4: Anatomy and physiology of the inner ear. (a) Schematic of a mammalian ear including the external, middle and inner ear, which contains the cochlea and vestibular end organs. The endoelectronics chip is illustrated in one possible location, although the experiments were done with the chip located outside of the middle ear cavity. (b) Cross-section of a typical cochlear half-turn, showing the endolymphatic space (yellow) bordered by tight junctions (red), the stria vascularis (green) and hair cells (blue), which are contacted by primary auditory neurons (orange) [5]. 
methods of direct transformation of glucose energy into electric energy, which operate according to the scheme of living organisms. It is absolutely vital since secretory products of such a transformer have to be the same as in a live cell. It will enable us to implant similar transformers directly into the human body not worrying about possible harmful discharge.

One of the variants of the chemical reaction sequence which takes place in adiabatic catalytic fuel cell operating on the glucose-oxygen reaction is the following [8]:

Anode: $\mathrm{C}_{6} \mathrm{H}_{12} \mathrm{O}_{6}+24 \mathrm{OH}^{-} \rightarrow 6 \mathrm{CO}_{2}+18 \mathrm{H}_{2} \mathrm{O}+24 \mathrm{e}^{-}$

Cathode: $6 \mathrm{O}_{2}+12 \mathrm{H}_{2} \mathrm{O}+24 \mathrm{e}^{-} \rightarrow 24 \mathrm{OH}$

Overall: $\mathrm{C}_{6} \mathrm{H}_{12} \mathrm{O}_{6}+6 \mathrm{O}_{2} \rightarrow 6 \mathrm{CO}_{2}+6 \mathrm{H}_{2} \mathrm{O}$

$$
\Delta \mathrm{H}^{0}: \quad 2: 870 \times 10^{6} \mathrm{Jmol}^{-1}
$$

$\mathrm{U}^{0}: 1.24 \mathrm{~V}$

As we can see, oxidation of one glucose molecule results in release of 24 electrons.

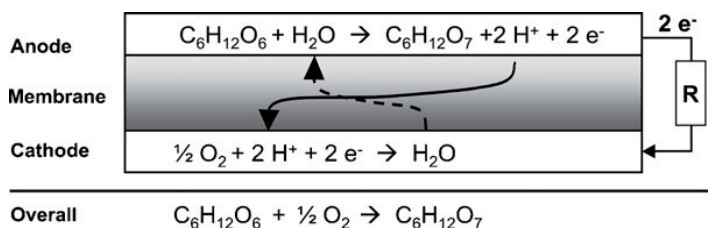

Fig. 5: General electrode reactions of an abiotically catalyzed glucose-oxygen fuel cell, assuming ahydroxyl ion conducting membrane and gluconic acid as the reaction product. According to Ref. [8].

The bio-battery implementing the reactions described above was set forth in work [6]. Provided the size of it is small, it ensures the power of

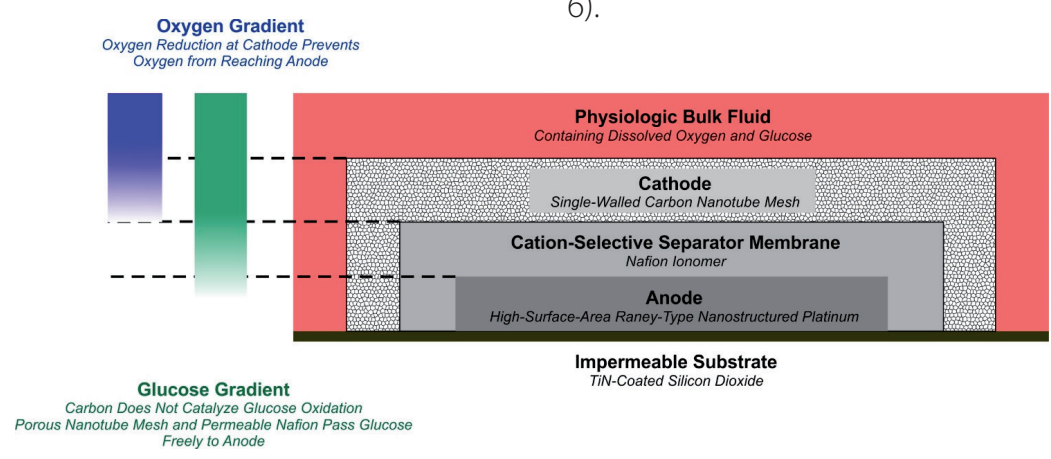

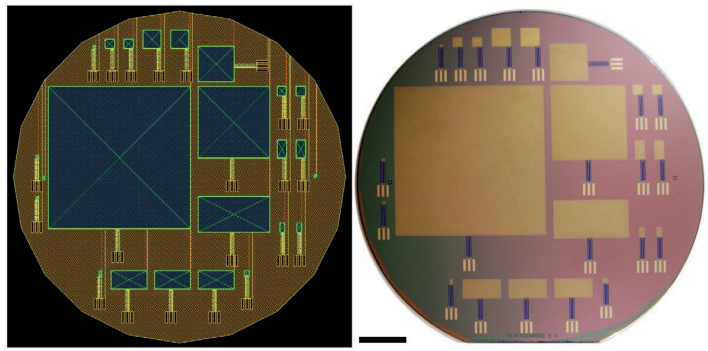

Fig. 7: Photolithography Masks and Fabricated Fuel Cells. The image at left shows a set of superimposed photolithographic masks for glucose fuel cells of various sizes, arranged for fabrication on a silicon wafer $150 \mathrm{~mm}$ (6 inches) in diameter. The largest device depicted has an anode that measures $64 \mathrm{~mm}$ by $64 \mathrm{~mm}$. The anodes of the other fuel cells shown are scaled-down versions of the large device, with length and width alternately reduced by factors of two. The schematic was constructed by overlaying the four process layers: yellow, platinum; orange, roughened platinum anode (aluminium deposition for annealing); blue, Nafion; green, cathode (single-walled carbon nanotubes in Nafion). The photograph at right shows the corresponding silicon wafer as fabricated. Scale Bar: $2 \mathrm{~cm}$ [7].

$3.4 \mu \mathrm{Watt} / \mathrm{cm}^{-2}$ (maximum $180 \mu \mathrm{Watt} / \mathrm{cm}^{-2}$ ) and has a very long life cycle. Modern semiconductor technology is used for its production, which enables development of combined implants with power management integrated circuit (PMIC) (for instance, on the opposite side of the microcircuit chip). Glucose is oxidized on the nano-structured surface of the activated platinum anode. The oxygen reconstructs to water on the surface, self-organizing single walled carbon nanotube meshes embedded into Nafion membrane, which forms cathode and is influenced by the biological environment (Figure 5, 6).

Fig. 6: Glucose Fuel Cell in Cross Section. This schematic cross-section of the glucose fuel cell illustrates the structure of the device, as well as the oxygen and glucose concentration gradients crucially associated with its cathode and anode half-cell reactions, and underlying their respective site specificity [7]. 

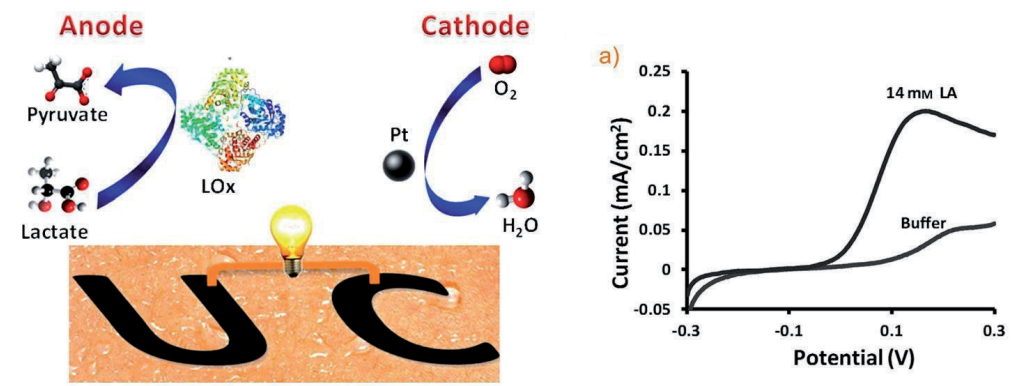

I) Anode ' $U$ '
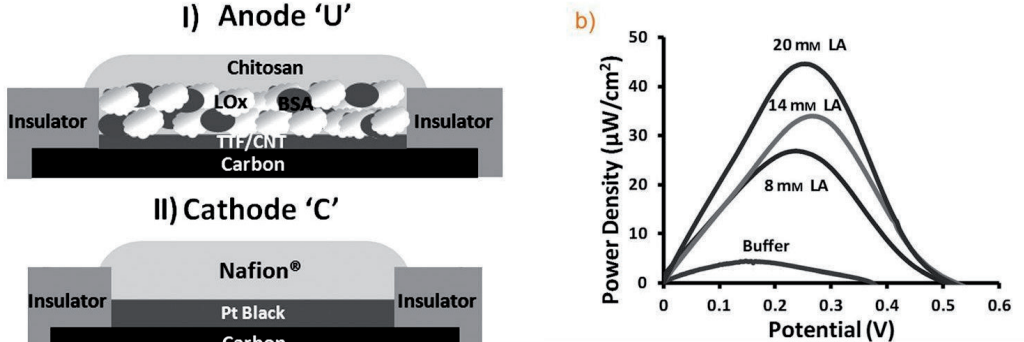

Fig. 8: On the left - IIlustration of the epidermal tBFC and the constituents of the anode (I) and cathode (II), on the right - a) Polarization curves for the CNT/TTF/LOX bio-anode in the absence and presence of lactate (14 mm) in 0.2m Mcllvaine buffer solution (pH 5.5). b) Power density of the $t B F C$ at varying lactate (LA) concentrations in $0.2 \mathrm{~m}$ Mcllvaine buffer solution ( $\mathrm{PH}$ 5.5). FAD=flavin adenine dinucleotide [9].

The drawback of the glucose-based batteries described above is the necessity to be implanted into the human body. It is quite acceptable when it comes to powering a hearing device or artificial retina, but the vast majority of the systems require non-invasive installation methods (not every person will agree to undergo surgical intervention in order to have a gadget installed). Therefore, there have been developed bio-batteries [9] implementing lactic acid (lactate) contained in human sweat. Practically the case in question is non-permanent bio-tattoo (tattooed on the skin of a person). The electrode structure as well as electrical parameters of the bio-tattoo is specified in Figure 8.

The capacity of this bio-battery amounts to 50 $\mu$ Watt. $\mathrm{cm}^{-2}$, which reduces by $10 \%$ after the first 14 days of operation and by $50 \%$ after 28 days of operation.

The major advantage of the glucose-based batteries in comparison with other current sources is its extremely simple charging method. To tell the truth, while there is enough glucose around such an element, the system does not need any charging (glucose is always available in human body). The main disadvantage of such elements is aging, i.e. reduction of their efficiency with time.

The most successful practical implementation of autonomous (non-implanted) sugar-based fuel cells is considered to be carried out by the company SONY, with their development having the dimensions $39 \times 39 \times 39 \mathrm{~mm}$ and the power of 50 mWatt. It is absolutely sufficient for powering, for instance, MP3 player [10].

\section{Conclusions}

The latest achievements in the field of microelectronics are characterized by diminutiveness, wide functional possibilities and low cost. Various multimedia devices such as smartphones, i-pads etc. are particularly popular. Such gadgets are powered on the basis of chemical elements that are harmful to the environment. On top of that, the capacity and amount of the energy cells are increasing from year to year. Taking into account the facts mentioned above, brand new types of energy sources based on biochemical effects are proposed to be implemented. Such power sources can be located directly on the surface of human body and with the help of clothes ("smart clothes" system) efficiently implement the energy emitted by human organism for powering various portable devices [11], primarily of medical character, restricted to official use, or just aimed at entertainment. 


\section{References and Notes}

[1] Gritsenko A., Nikiforov V., Shchegolev T., Status and prospects of development of piezoelectric generators, "Components and technologies", FineStreet, Russia: St. Petersburg, no9, 2012, p. 63-68.

[2] Starner T., \& Paradiso A. Human Generated Power for Mobile Electronics, Georgia Tech, Atlanta, GA, USA, 2005.

[3] Sue, C. H., and N. C. Tsai., Human powered MEMS-based energy harvest devices, Appl. Energy, 2011, 93:390-403.

[4] Riemer, R., and A. Shapiro., Biomechanical energy harvesting from human motion: theory, state of the art, design guidelines and future directions, J. Neuroeng. Rehabil., 2011, 8:1-13.

[5] Patrick P Mercier, Andrew C Lysaght, Saurav Bandyopadhyay, Anantha P Chandrakasan, Konstantina M Stankovic, Energy extraction from the biologic battery in the inner ear, Nature Biotechnology, Vol. 30, No. 12, 2012, p.1240-1243.

[6] Zhiguang Zhu, Tsz Kin Tam, Fangfang Sun, Chun You, Y.-H. Percival Zhang. A high-energy density sugar biobattery based on a synthetic enzymatic pathway, Nature Communications 5, Article number:
3026,2014 , p. 5:3026.

[7] Rapoport Benjamin I., Jakub T. Kedzierski, Rahul Sarpeshkar. A glucose fuel cell for implantable brain-machine interfaces, PLoS ONE 7(6), 2012, p. e38436:1-15.

[8] Kerzenmacher S, Ducre'e J, Zengerle R, von Stetten F. Energy harvesting by implantable abiotically catalyzed glucose fuel cells. Journal of Power Sources, Vol. 182(1), 2008, p. 1-17.

[9] Wenzhao Jia, Gabriela Valdés-Ramírez, Amay J. Bandodkar, Joshua R. Windmiller, Joseph Wang, Epidermal Biofuel Cells: Energy Harvesting from Human Perspiration, Angewandte Chemie Internationa Edition English, Vol. 52, Issue 28, 2013, p. 7233-7236

[10] Sony corporation. http://www.sony.net/Sonylnfo/News/ Press/200708/07-074E/

[11] Alexander Molnar, Vitaly Gerasimov, Igor Piotr Kurytnik. The development of monitoring devices in the system "man-clothes" based on modern microcontrollers, Pomiary Automatyka Robotyka, R. 20, no 2. 2016, p. 31-35.

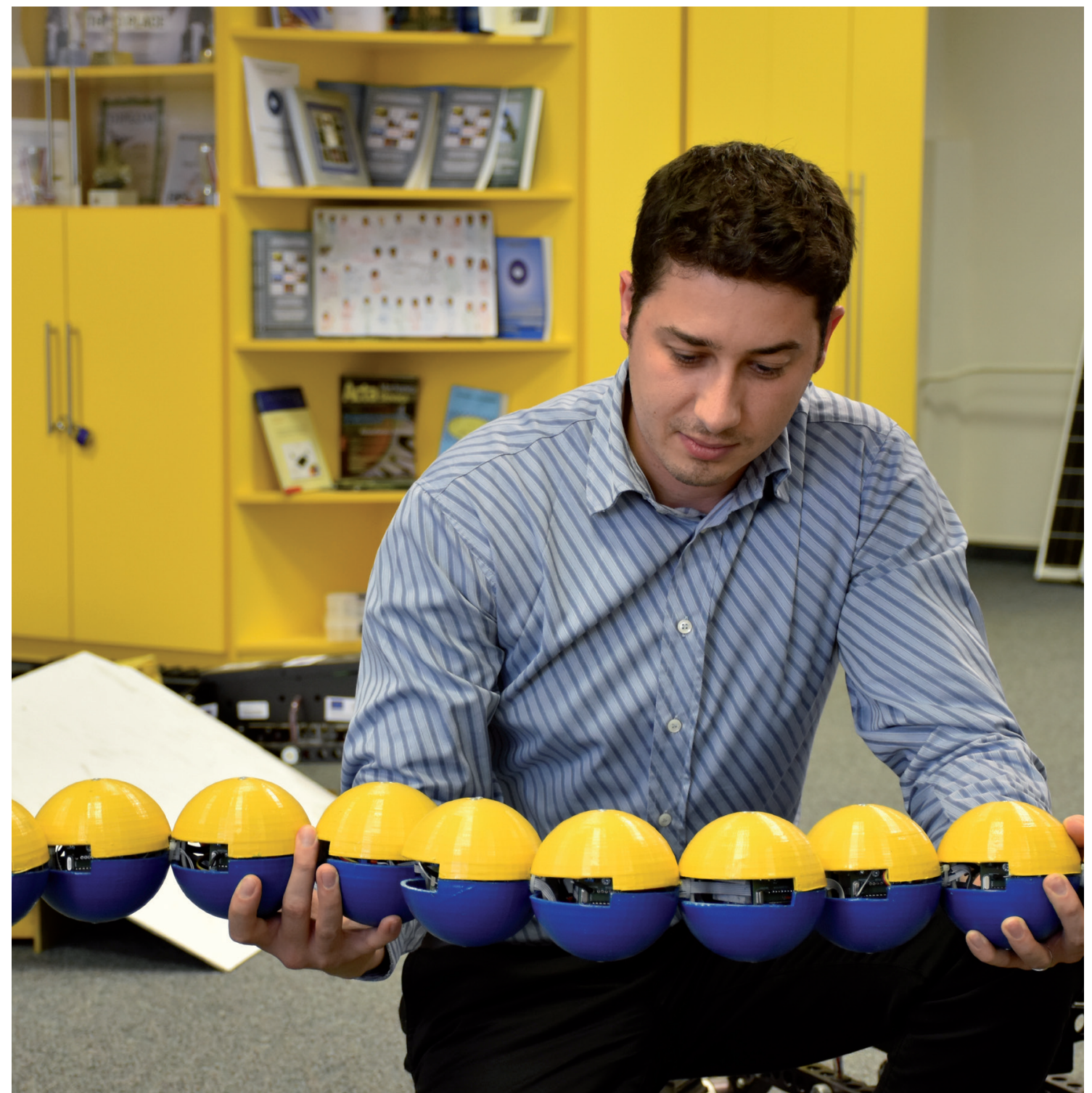

